On Equationally Compact Semilattices by

Sydney Bulman-Fleming

Nr. 24

1972 


\title{
On Equationally Compact Semilattices
}

by

\author{
Sydney Bulman-Fleming
}

Although equationally compact semilattices have been completely characterized [4], the question of J. Mycielski "Is every equationally compact semilattice the retract of a compact topological semilattice?" (first stated in [5] for general algebras, and posed anew in [7] for semilattices) has heretofore remained unanswered. The main purpose of the present paper is to provide an affirmative answer to this question.

Further, a new notion of "algebraic" compactness is introduced which among all semilattices singles out exactly those in which every chain is finite. Such semilattices are in turn compact topological ones in view of the more general result that the class of compact topological semilattices includes all join-complete semilattices in which every chain has a least element.

Throughout this paper the term "semilattice" shall mean "join semilattice".

The results presented here form a part of the author's doctoral thesis. For inspiration and guidance during the course of this investigation the author expresses gratitude to G.H. Wenzel.

\section{\$1. Preliminaries}

An algebra $O=\langle A ; F\rangle$ (see [3]) is called equationally compact if the existence of a simultaneous solution of every finite subset $\Sigma$ ' of anset $\Sigma$ of polynomial equations with constants in $S$ implies the existence of a simultaneous solution of $\Sigma$. (Detailed discussions of equational compactness can be found, for example, in [9], [10] and [11].) The equationally compact semilattices $\gamma=\langle s ; V\rangle$ were 
characterized in [4] as those which satisfy the following three conditions:

(C1) The supremum of any non-empty subset $\mathrm{X} \subseteq \mathrm{S}$ exists; i.e. $\gamma$ is join-complete.

(C2) The infimum of any downward-directed subset $\mathrm{D} \subseteq \mathrm{S}$ exists.

(C3) If $a \in S$, and if $D \subseteq S$ is a downward-directed subset of $S$, then $a \vee(\wedge D)=\wedge(a \vee d \mid d \in D)$.

\section{Definition 1.1}

The interval topology of a partially ordered set $P$ $=\langle P ; \leqslant\rangle$ is defined by taking as a sub-basis of closed. sets the collection of all intervals $(-\infty, a]=\{p \in P \mid p \leqslant a\}$ and $[a, \infty)=\{p \in P \mid p \geqslant a\}$ as a ranges over $P$.

A fundamental result of 0 . Frink [2] states that a lattice is compact in its interval topology iff it is complete. Application of Frink's methods yields that a semilattice $\gamma=\langle S ; V\rangle$ is compact in its interval topology iff conditions (C1) and (C2) above are satisfied. Thus, every equationally compact semilattice is compact in its interval topology (although the topology is not, in general, Hausdorff).

An algebra $O=\langle A ; F\rangle$ is called a (compact) $\mathcal{J}$-topological. algebra if $J$ is a (compact) Hausdorff topology on $A$ such that if $f \in F$ and $f: A^{n} \longrightarrow A$, then $f$ is a continuous mapping if $A^{n}$ is endowed with the product topology. An algebra which is known to support a (compact) Hausdorff topology with respect to which all its fundamental operations are continuous is called simply a (compact) topological algebra if no particular topology $\mathrm{J}$ is specified.

A subalgebra $\sigma_{\text {of }}$ an algebra $\mathscr{L}$ is called a retract of $\mathscr{L}$ if there exists a homomorphism $\mathrm{p}$ from $\mathcal{L}$ onto $\boldsymbol{O}$. which acts as the identity function on $\sigma$. Such a homomorphism $p$ is called a retraction of $\mathscr{L}$ onto $\boldsymbol{O}$. 
Definition 1.2

Let $\mathcal{X}=\langle L ; \vee, \Lambda\rangle$ be a complete lattice, and let $\left(x_{\alpha}\right)_{\alpha \in D}$ be a net in $L$. The net $\left(x_{\alpha}\right)_{\alpha \in D}$ is said to order converge to $x \in L$ iff $V\left(\Lambda\left(x_{\alpha} \mid \alpha \geqslant \gamma\right) \mid \gamma \in D\right)=x=$ $\wedge\left(V\left(x_{\alpha} \mid \alpha \geqslant \gamma\right) \mid \gamma \in D\right)$. The order topology of $\mathcal{L}$ is defined as follows: $F \subseteq L$ is closed in the order topology of $\mathscr{L}$ iff whenever a net $\left(x_{\alpha}\right)_{\alpha \in D}$ in $F$ order converges to $x \in L$, then. $x \in F$.

It is well-known that, in general, order convergence does not coincide with convergence in the order topology. However, should $\mathcal{X}$ be a complete and completely distributive ${ }^{1}$ lattice, then in fact the following three statements are equivalent, where $\left(x_{\alpha}\right)_{\alpha \in D}$ is a net in $L$ and $x \in L$ :

(01) $\left(x_{\alpha}\right)_{\alpha \in D}$ converges to $x$ in the interval
topology. topology .

(02) $\left(x_{\alpha}\right)_{\alpha \in D}$ converges to $x$ in the order

(03) $\left(x_{\alpha}\right)_{\alpha \in D}$ order converges to $x$.

Moreover, if $\mathscr{L}$ is a complete and completely distributive lattice, then the interval topology (which coincides with the order topology) is compact, Hausdorff, and makes both lattice operations continuous. Most of the observations of this paragraph are well-kno $n$ and can be found, for example, in [6]. They will always in this paper be applied to the Boolean lattice $\left\langle 2^{\mathrm{S}} ; U, \cap\right\rangle$ of all subsets of a set $S$.

$1_{\text {A }}$ complete lattice $\mathscr{L}$ is called completely distributive iff the following condition holds: for every doubly-indexed set $\left\{x_{i, j} \mid i \in I, j \in J_{i}\right\}$ of elements of $L$, the equation $V\left(\wedge\left(x_{i, j} \mid j \in J_{i}\right) \mid i \in I\right)=\Lambda\left(V\left(x_{i, \varphi(i)}\right) i \in I\right) \mid \varphi \in T\left(J_{i} \mid\right.$ $i \in I))$ and its dual are satisfied. 
This section concludes with an embedding lemma which will play a crucial role in the remainder of this paper.

\section{Lemma 1.3}

Let $\gamma=\langle S ; V\rangle$ be a semilattice. For each element $s \in S$ let $e(s)=\{t \in S \mid s \leqslant t\} \in 2^{S}$. Then $e$ is an embedding of $\gamma$ into $2^{\gamma}=\left\langle 2^{S}\right.$; $\left.U\right\rangle$ such that if $X \subseteq S$ and $V X=s$, then $e(s)=\bigcup(e(x) \mid x \in X)$.

Proof:

Only the fact that e preserves suprema will be proven. If $V x=s$, then for any element $t \in S$, $t \in e(s)$ iff $t \neq s$. But this is equivalent to $t \neq x$ holding for some $x \in X$, or in other words to $t$ belonging to $U(e(x) \mid x \in X)$.

\section{\$2. Mycielski's Question for Semilattices}

J. Mycielski observed [5] that every retract of a compact topological algebra is equationally compact. To his question "Is every equationally compact algebra retract of a compact topological algebra?" affirmative answers were given in a large number of specific equational classes (for a summary of these results see [11]) but that the question in its general setting has a negative answer was shown by. W. Taylor [8], who by a graph-theoretical method exhibited an equationally compact algebra of type $\langle 1 ; 1\rangle$ which is retract of no compact topological algebra. As mentioned in the introduction, the problem is attacked for the class of semilattices in the present paper.

At the outset, it should be remarked that the topology of a compact topological semilattice is a-compatible in the sense of E.E. Floyd [1]. Since Floyd. was able to find a compiete Boolean algebra (which can in particular be considered an equationally compact semilattice) with no Hausdorff and $\sigma$-compatible topology, the problem of Mycielski cannot admit the solution that every equationally compact semilattice is itself a compact topological one. 
A solution for Mycielski's problem for the class of semilattices is now given.

\section{Lemma $\underline{2.1}$}

Let $P$ and $Q$ be partially ordered sets and let $f: P \rightarrow Q$ be a mapping which satisfies $p_{1} \leqslant p_{2}$ iff $f\left(p_{1}\right) \leqslant f\left(p_{2}\right)$, for all $p_{1}, p_{2} \in P$. Let $X$ and $Y$ be subsets of $P$ such that $\Lambda \mathrm{X}, \Lambda \mathrm{Y}, \Lambda \mathrm{f}(\mathrm{X})$ and $\Lambda \mathrm{f}(\mathrm{Y})$ all exist.

Then $\Lambda f(X)=\Lambda f(Y)$ implies $\Lambda X=\Lambda Y$.

\section{Proof:}

Suppose the hypotheses are all satisfied. Let $w=$ $\Lambda X$ and $z=\Lambda Y$. If $w \neq y_{0}$ for some $y_{0} \in Y$, then $f(w) \notin$ $f\left(y_{0}\right)$. But $f(w) \leqslant \Lambda f(x)$ since $w$ is a lower bound for $x$ and $f$ preserves order. Therefore $\Lambda \mathrm{f}(\mathrm{X}) \leqslant \mathrm{f}\left(\mathrm{y}_{0}\right)$, which gives the contradiction $\Lambda f(Y) \$ f\left(y_{0}\right)$.

Consequently, $w$ is a lower bound for $Y$, so $w \leqslant z$. Similarly $\mathrm{z} \leqslant \mathrm{w}$ and the proof is completed.

Corollary 2.2

Let $\gamma=\langle S ; V\rangle$ be a semilattice, let $2^{\gamma}=\left\langle 2^{S} ; U\right\rangle$ and let $e$ be the embedding of 1.3. Let $X$ and $Y$ be subsets of $S$ such that $\Lambda X$ and $\Lambda Y$ both exist. Then $\cap(e(x) \mid x \in X)$ $=\bigcap(e(y) \mid y \in Y)$ implies $\Lambda X=\Lambda Y$.

\section{Theorem 2.3}

Let $\gamma=\langle S ; V\rangle$ be a semilattice. Then $\gamma$ is equationally compact iff $\gamma$ is retract of a compact topological semilattice.

Proof:

It was remarked at the beginning of this section that retracts of compact topological algebras are equationally compact.

Assume therefore that $\gamma$ is equationally compact. Let $R=e(S)$ where $e$ is the embedding of 1.3 , let $R=\langle R$; U $\rangle$ be the corresponding subsemilattice of $2^{\gamma}$ (which is of course isomorphic to $\boldsymbol{\gamma}$ ) and let $\bar{R}=\langle\bar{R} ; U\rangle$, where $\bar{R}$ denotes the closure of $R$ in the interval topology (= order topology = Tychonoff topology) of $2^{\gamma}$. Thus $\bar{R}$ is a compact topological 
semilattice when given the relative topology. The theorem will be proven if a homomorphism $\mathrm{p}: \overline{\boldsymbol{R}} \longrightarrow \gamma$ can be given satisfying $p(e(s))=s$ for all $s \in S$.

To accomplish this task, observe that for any $x \in \bar{R}$ there exists a downward-directed subset $M \subseteq S$ such that $x=\bigcap(e(m) \mid m \in M)$. For, $x$ is the order limit of some net $\left(e\left(x_{\alpha}\right)\right)_{\alpha \in D}$ in $R$ (by the equivalence of statements (01)(03) above). For each $\gamma \in D$ define $m_{\gamma}=V\left(x_{\alpha} \mid \alpha \geqslant \gamma\right)$. Then the set $M=\left\{m_{\gamma} \mid \gamma \in D\right\}$ is downward-directed and because $e$ preserves suprema one finds that $x=\bigcap(e(m) \mid m \in M)$.

Should $x \in \bar{R}$ admit two representations $x=\bigcap(e(m) \mid m \in M)$ and $x=\bigcap(e(n) \mid n \in N)$ where $M$ and $N$ are downward-directed subsets of $S$, then $\Lambda M=\Lambda N$ by 2.2. Thus $p(x)$ can be unambiguously defined as $\Lambda \mathrm{M}$, where $M$ is any downwarddirected subset of $S$ for which $x=\bigcap(e(m) \mid m \in M)$.

It is clear that $\mathrm{p}(e(s))=s$ for any $s \in S$. That $p$ is a homomorphism, finally, is seen as follows. Let $x$, $y \in \bar{R}$ and suppose $M$ and $N$ are downward-directed subsets of $S$ such that $x=\bigcap(e(m) \mid m \in M)$ and $y=\bigcap(e(n) \mid n \in N)$. Then $p(x) \vee p(y)=(\Lambda M) \vee(\Lambda N)=\Lambda(m \vee n \mid m \in M, n \in N)$ by condition (C3). On the other hand, $x U_{y}=\bigcap(e(m \vee n) \mid m \in M$, $n \in N)$, and since $\{m \vee n \mid m \in M, n \in N\}$ is a downward-directed subset of $S$ affording a representation of $x U y$ as prescribed in the definition of $p$, the desired result $p(x \cup y)=$ $\Lambda(m \vee n \mid m \in M, n \in N)$ is obtained.

\section{\$3. Further Results}

To begin this section, a new form of algebraic compactness is defined which will be studied in detail for the class of semilattices.

\section{Definition 3.1}

Let $K$ be a class of algebras of the same type and let $\boldsymbol{a}$ be an algebra in $\mathrm{K}$. Then $\boldsymbol{a}$ is called $\mathrm{K}$-compact iff for every algebra $\mathscr{W}$ in $K$ such that $\boldsymbol{O}$ is a subalgebra of $\mathcal{L}$ the following condition is satisfied: the existence 
of a simultaneous solution in $\sigma$ of every finite subset $\Sigma$ ' of ayset $\Sigma$ of polynomial equations with constants in B implies the existence of a simultaneous solution of $\Sigma$ in $\boldsymbol{\theta}$.

One observes that if $K_{2}$ is a class of algebras of the same type, if $K_{1} \subseteq K_{2}$, and if $\mathcal{G}$ is an algebra in $K_{1}$,

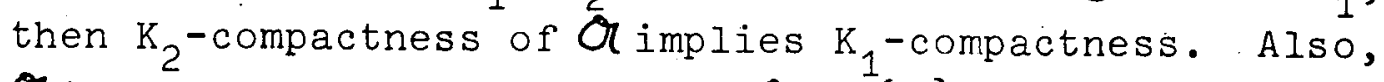
$\boldsymbol{O}$ is equationally compact iff $\boldsymbol{O}$ is $\{\boldsymbol{O}\}$-compact, so if $\boldsymbol{O}$ is $\mathrm{K}$-compact for any class $\mathrm{K}$, then it is equationally compact.

It is well-known that all compact topological algebras, so in particular all finite ones, are equationally compact. The following proposition shows that a finite algebra is in fact $\mathrm{K}$-compact for any class $\mathrm{K}$ to which it belongs. Although the proof is topological in nature, it becomes apparent in light of the characterization theorem of $\mathrm{K}$-. compact semilattices which follows that in general compact topological algebras may fail to be K-compact.

Proposition 3.2

Let $K$ be a class of algebras of the same type and let $\boldsymbol{\theta}=\langle A ; F\rangle$ be a finite algebra in $K$. Then $\boldsymbol{Q}_{\text {is } \mathrm{K} \text {-compact. }}$ Proof:

Let $\mathscr{L}=\langle B ; F\rangle \in K$ be any extension of $\boldsymbol{C}$. Then any polynomial $p$ in the variables $\left(x_{\gamma}\right)_{\gamma<\alpha}$ with constants in $B$ can be considered a continuous mapping from $A^{\alpha}$ into $B$, where $B$ is given the discrete topology and $A^{\alpha}$ is endowed with the relative topology $\mathcal{J}$ induced from the product topology of $B^{\alpha}$. $\mathcal{J}$ is in fact a compact topology since it is simply the $\alpha$-fold product of the discrete topology on A. Thus (because $B$ is Hausdorff) any polynomial equation $p=q$ with constants in $B$ has a closed solution set in $A^{\alpha}$ and so the compactness of $\mathcal{G}$ assures that any set of polynomial equations with constants in $B$ finitely solvable in $\boldsymbol{G}$ is in fact solvable in $\boldsymbol{a}$.

To initiate the study of $\mathrm{K}$-compact semilattices (for 
$K$ to be specified later) the following sequence of lemmas is given. It is noted that Lemmas $3.4,3.5$ and one part of the proof of Theorem 3.6 are modelled after similar arguments in [4].

\section{Lemma 3.3}

Let $\boldsymbol{Q}=\langle\mathrm{A} ; \mathrm{V}\rangle$ be a semilattice in which every chain has a largest element. If $\phi \neq X \subseteq A$ then there exists a finite subset $B \subseteq X$ such that $V X=V B$.

\section{Proof:}

Let $\mathrm{T}=\{V \mathrm{~B} \mid \phi \neq \mathrm{B} \subseteq \mathrm{X}$ and $\mathrm{B}$ is finite $\}$. Then $\mathrm{T}$ is directed and so contains a greatest element $V B$. clearly $\vee B=V X$.

Lemma 3.4

Let $\gamma=\langle S ; V\rangle$ be a semilattice in which every chain has a largest element, suppose $\gamma$ is a subsemilattice of the semilattice $\boldsymbol{\sigma}=\langle\mathrm{A} ; \boldsymbol{V}\rangle$, and let $\mathrm{K} \subseteq \mathrm{S}^{\alpha}$ be a set of solutions of the equation $a \vee x_{i_{0}} \vee \ldots \vee x_{i_{n-1}}=b \vee x_{j_{0}} \vee \ldots$ $V x_{j-1}$ where $a, b \in A ; \alpha$ is an ordinal, and $i_{0}, \ldots, i_{n-1}$, $j_{0}, \ldots, j_{m-1}$ are elements of the $\operatorname{set}\{\gamma \mid \gamma<\alpha\}$. Then $t=$ $V K$ (the supremum being taken in the semilattice $\gamma^{\alpha}$ ) is a solution of the equation $a V x_{i_{0}} \vee \ldots V x_{i_{n-1}}=b \vee x_{j_{0}} V \ldots$ $\bigvee x_{j-1}$

\section{Proof:}

Let $k_{0}, \ldots, k_{r-1}$ be the distinct members of the set $\left\{i_{0}, \ldots, i_{n-1}, j_{0}, \ldots, j_{m-1}\right\}$ and let $J=\left\{k_{0}, \ldots, k_{r-1}\right\}$. Define $K_{1} \subseteq S^{J}$ as follows: $f \in K_{1}$ iff there exists $g \in K$ such that $g\left(k_{i}\right)=f\left(k_{i}\right)$ for all $k_{i} \in J$. The semilattice $\sigma^{J}$ has the property that every chain has a largest element. Hence by 3.3 there exist $b_{0}, \ldots$, $b_{s-1} \in K_{1}$ such, that $u=V K_{1}=b_{0} V \ldots V b_{s-1}$. Clearly $u\left(k_{i}\right)=t\left(k_{i}\right)$ for all $k_{i} \in J$. Thus, $a \vee t\left(i_{0}\right) \vee \ldots V t\left(i_{n-1}\right)$ $=a \vee u\left(i_{0}\right) V \ldots V u\left(i_{n-1}\right)=a V b_{0}\left(i_{0}\right) V \ldots V b_{s-1}\left(i_{0}\right) V \ldots$ 
$V b_{0}\left(i_{n-1}\right) V \ldots V b_{s-1}\left(i_{n-1}\right)=b V b_{0}\left(i_{0}\right) \vee \ldots V b_{s-1}\left(i_{n-1}\right)$ $=b \vee t\left(i_{0}\right) \vee \ldots V t\left(i_{n-1}\right)$, so $t$ is a solution of the equation.

\section{Lemma $\underline{3.5}$}

Let $\gamma=\langle s ; V\rangle$ be a semilattice in which every chain has a smallest element, suppose $\gamma$ is a subsemilattice of the semilattice $\theta=\langle A ; V\rangle$, and let $K \subseteq S^{\alpha}$ be a downwarddirected set of solutions of the equation $a \vee x_{i_{0}} \vee \ldots \vee x_{i_{n-1}}$

$=b \vee x_{j_{0}} \vee \ldots V x_{j_{m-1}}$ where $a, b \in A, \alpha$ is an ordinal, and $i_{0}$, $\ldots, i_{n-1}, j_{0}, \ldots, j_{m-1}$ belong to the set $\{\gamma \mid \gamma<\alpha\}$. Then $t$ $=\bigwedge \mathrm{K}$ (the infimum being taken in the semilattice $\tau^{\alpha}$ ) is a solution of the equation $a \vee x_{i_{0}} \vee \ldots V x_{i_{n-1}}=b \vee x_{j_{0}} \vee \ldots$ $V \mathrm{x}_{j_{\mathrm{m}-1}}$

\section{Proof:}

Let $J$ and $K_{1}$ be defined as in the proof of 3.4. Let $v$ be the least element of $k_{1}$. Then $v\left(k_{i}\right)=t\left(k_{i}\right)$ for each $k_{i} \in J$ and $t$ satisfies the required equation since there exists $g \in K$ such that $g\left(k_{j}\right)=v\left(k_{i}\right)$ for all $k_{i} \in J$.

In the subsequent discussion, $\mathbb{\$}$ denotes the class of all semilattices and $\mathbb{L}_{S}$ denotes the class of all. semilattices which are join-semilattices of lattices. In other words, $\mathbb{L}_{\mathrm{S}}$ consists of those semilattices $\mathcal{L}$ $=\langle L ; V\rangle$ such that the corresponding partial order is a lattice order. Clearly $\mathbb{L}_{S} \subseteq \$$.

Theorem 3.6

Let $\gamma=\langle S ; V\rangle$ be a semilattice. Then the following conditions are equivalent:

(K1) $\gamma$ is $\boldsymbol{\gamma}$-compact.

(K2) $\boldsymbol{\gamma}$ is $\mathbb{L}_{S^{- \text {compact. }}}$

(K3) $\gamma$ contains no infinite chains. 


\section{Proof:}

The equivalence of (K1) and (K2) is an easy consequence of the fact that any partially ordered set can be embedded in a lattice with preservation of all existing suprema and infima (for example, via the Dedekind-McNeille embedding).

To show (K3) implies (K1) let $\dot{\sigma}=\langle A ; V\rangle$ be any semilattice extension of $\gamma$ and let $\Sigma=\left\{\sigma_{i} \mid i \in I\right\}$ be a set of polynomial equations with constants in $\mathrm{A}$, involving the variables $\left\{x_{\gamma} \mid \gamma<\alpha\right\}$, such that each finite subset of $\Sigma$ has a solution in $\gamma$. For each finite subset $\Sigma^{\prime}$ of $\Sigma$ let $K\left(\Sigma^{9}\right) \subseteq S^{\alpha}$ be the set of solutions of $\Sigma^{\prime}$. By 3.4 , $t\left(\Sigma^{\prime}\right)=V K\left(\Sigma^{\prime}\right)$ is also a solution of $\Sigma^{\prime}$. For each $i \in I$ let $J_{i}=\left\{t\left(\Sigma^{\prime}\right) \mid \Sigma^{\prime}\right.$ is a finite subset of $\Sigma$ and $\left.\sigma_{i} \in \Sigma^{\prime}\right\}$. Then $J_{i}$ is a downward-directed set of solutions of $\sigma_{i}$ and so $\wedge^{i}$ by 3.5 is also a solution of $\sigma_{i}$. since ${ }^{i} J_{i}$ and $J_{j}$ are mutually co-initial for all $i, j \in I$ the infimum of any $J_{i}$ is a solution of $\boldsymbol{\Sigma}_{i}$.

Finally to show (K1) implies (K3) suppose $\boldsymbol{\gamma}$ is $\boldsymbol{\$}$ compact (and hence equationaliy compact) but contains an infinite chain. Then either (a) $\gamma$ contains a chain $\boldsymbol{b}_{1}$ with no largest element, or (b) $\gamma$ contains a chain $b_{2}^{1}$ with no smallest element. In case (a) let $z=V \sigma_{1}$, let $A_{1}=S \dot{U}\{w\}$ and extend the partial order on $\gamma$ to a partial order on $A_{1}$ by defining $x \geqslant w$ iff $x=w$ or $x \geqslant z$, and $x \leqslant w$ iff $x=w$ or $x \leqslant c$ for some $c \in G_{1}$. Embed $\left\langle A_{1} ; \leqslant\right\rangle$ into a lattice $\mathcal{L}=\langle L ; \vee, \wedge\rangle$ in such a way that suprema and infima are preserved, and let $\boldsymbol{O}$ be the semilattice $\langle I ; v\rangle$. Then the set of equations $\Sigma=\left\{x \vee c=x \mid c \in G_{1}\right\} \cup\{x \vee w=w\}$ is finitely solvable in $\gamma$, but not solvable in $\gamma$. Case.(b) is treated entirely similarly. In either case the $\boldsymbol{S}$ compactness of $\gamma$ is contradicted.

In conclusion, a large class of compact topological semilattices will be delineated, a subclass of which is the class of $\boldsymbol{\$}$-compact semilattices characterized above.

Theorem 3.7

Let $\gamma$ be a join-complete semilattice (i.e. $\gamma$ satisfies 
condition (C1) previously stated) in which every chain has a least element. Then $\gamma$ is a compact topological semilattice.

Proof:

Let $\gamma$ be embedded in $2^{\gamma}=\left\langle 2^{S}\right.$; U v via the embedding e of 1.3. If $g$ is the topology e(S) inherits as a subspace of $2^{S}$, where the latter as usual is given its interval topology, then clearly $e(\gamma)$ is a $\}$-topological semilattice. Under the present hypotheses, moreover, $I$ is compact.

To verify the latter statement it suffices by the Sub-base Theorem of Alexander to show that any family I of sub-basic $J$-closed subsets of $e(S)$ with the finite intersection property has a non-empty intersection. Accordingly let $\}=\left\{\left[X_{i}, Y_{i}\right] \cap e(S) \mid i \in I\right\}$ be such a family, where for each $i \in I X_{i}$ and $Y_{i}$ are subsets of $S$ with $X_{i} \subseteq Y_{i}$, and $\left[X_{i}, Y_{i}\right]=\left\{C \subseteq S \mid X_{i} \subseteq C \subseteq Y_{i}\right\}$.

Let $P=\{P \subseteq I \mid P$ is finite $\}$, for each $i \in I$ and $P \in P$ define $A_{i, P}=\left\{s \in S \mid X_{i} \subseteq e(s) \subseteq \bigcap\left(Y_{j} \mid j \in P\right)\right\}$, and for each $P \in P$ let $A_{P}=U\left(A_{i, p} \mid i \in I\right)$. Observe that each $A_{i, P}$ is non-empty. Finally, for each $P \in P$ let $s_{P}=V A_{P}$, and let $J=\left\{s_{P} \mid P \in P\right\}$.

If $P \in P$, it is clear that $U\left(X_{i} \mid i \in I\right) \subseteq e\left(s_{P}\right)$, since for each $i \in I$ there exists an element $s_{i, P} \in A_{i, P}$ and so $x_{i} \subseteq e\left(s_{i, p}\right)$ implies $U\left(x_{i} \mid i \in I\right) \subseteq U\left(e\left(s_{i, p}\right) \mid i \in I\right) \subseteq e\left(s_{P}\right)$.

Since $J$ is a downward-directed subset of $S$ it has a least element $s_{Q}$, where $Q \in P$. The considerations of the preceding paragraph yield at once the inclusion $U\left(x_{i} \mid i \in I\right)$ $\subseteq e\left(s_{Q}\right)$. On the other hand, for each $i \in I$ let $P_{i}=\{i\}$ $\in P$. Since $s_{Q} \leqslant s_{P_{i}}$ for all $i \in I$ it follows that $e\left(s_{Q}\right)$ $\subseteq \bigcap\left(Y_{i} \mid i \in I\right)$, and $\dot{s}_{0} \mathrm{e}\left(\mathrm{s}_{Q}\right) \in \bigcap \mathcal{F}$, which completes the proof of the theorem.

Corollary 3.8

Every $\sqrt{ }$-compact semilattice is a compact topological semilattice.

The procedure of 3.7 cannot in general be applied to semilattices with chains without smallest elements, as 
the following example shows.

\section{Example 3.9}

Let $C$ denote the set of negative integers, let $w$ denote a new element, and let $\gamma$ be the semilattice $\langle s ; v\rangle$, where $S=C \dot{U}\{w\}$, the elements of $C$ retain their usual order, and $w$ is smaller than any element of $C$. Then the topology on $e(S)$ inherited from $2^{\gamma}$ is not compact, since $\{w\}$ belongs to the set $\overline{e(S)} \backslash e(S)$. The situation is illustrated in the following diagram, where closed dots denote elements of $e(S)$ and open ones elements of $2^{S} \backslash e(S)$.

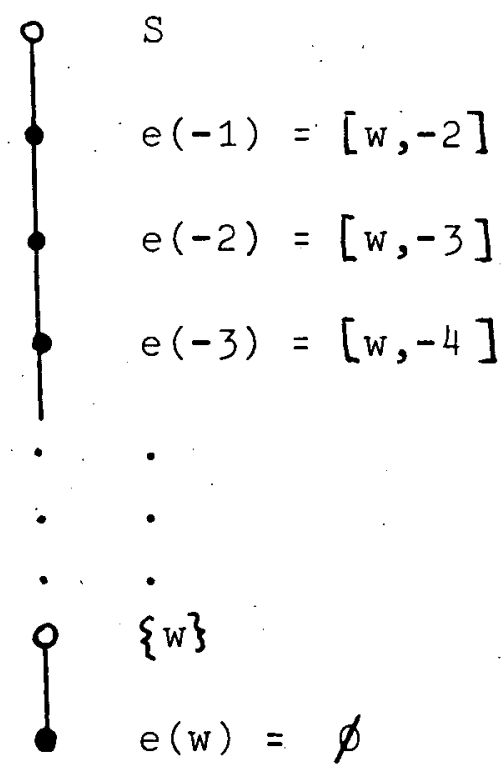

\section{Figure 1}

Observe that, while the method of Theorem 3.7 fails to equip the semilattice $\gamma$ of Example 3.9 with a compact topology, $\boldsymbol{\gamma}$ is nevertheless a compact topological semilattice in its own interval topology. 


\section{References}

[1] Floyd, E.E. Boolean Algebras with Pathological Order Topologies. Pac. J. Math. $\underline{5}$ (1955), 687-689.

[2] Frink, 0. Topology in Lattices. Trans. Amer. Math. Soc. 51 (1942), 569-582.

[3] Grätzer, G. Universal Algebra. Van Nostrand. Princeton, N.J. 1968.

[4] -.-.-- and Lakser, H. Equationally Compact Semilattices. Colloq. Math. 20 (1969), 27-30.

[5] Mycielski, J. Some Compactifications of General Algebras. Colloq. Math. 13 (1964), 1-9.

[6] Strauss, D.P. Topological Lattices. Proc. London Math. Soc. (3) 18 (1968), 217-230.

[ 7] Taylor, W. Convergence in Relational Structures. Math. Ann. 186 (1970), 215-227.

[8] ---.--- Atomic Compactness and Graph Theory. Fund. Math. 65 (1969), 139-145.

[9] -...-.-. Some Constructions of Compact Algebras. Annals of Math. Logic (to appear).

[10] Weglorz, B. Equationally Compact Algebras I. Fund. Math. 59 (1966), 289-298.

[11] Wenzel, G.H. Equational Compactness in Universal Algebras. Manuskripte, Fakultät für Mathematik und Informatik der Universität Mannheim, $\underline{68}$ Mannheim, W-Germany. Nr. 8, 1971. 